\title{
Deep optical observations of the central X-ray source in the Puppis A supernova remnant ${ }^{\star}$ (Research Note)
}

\author{
R. P. Mignani ${ }^{1}$, A. De Luca $^{2}$, S. Mereghetti ${ }^{2}$, and P. A. Caraveo ${ }^{2}$ \\ 1 Mullard Space Science Laboratory, University College London, Holmbury St. Mary, Dorking, Surrey, RH5 6NT, UK \\ e-mail: rm2@mssl.ucl.ac.uk \\ 2 INAF, Istituto di Astrofisica Spaziale, via Bassini 15, Milan, 20133, Italy
}

Received 12 November 2008 / Accepted 3 April 2009

ABSTRACT

\begin{abstract}
Context. X-ray observations revealed a group of radio-silent isolated neutron stars (INSs) at the centre of young supernova remnants (SNRs), dubbed central compact objects or CCOs, with properties different from those of classical rotation-powered pulsars. In at least three cases, evidence points towards CCOs being low-magnetized INSs, born with slow rotation periods, and possibly accreting from a debris disc of material formed out of the supernova event. Understanding the origin of the diversity of the CCOs can shed light on supernova explosion and neutron star formation models. Optical/infrared (IR) observations are crucial to test different CCO interpretations.

Aims. The aim of our work is to perform a deep optical investigation of the CCO RX J0822.0-4300 in the Puppis A SNR, one of the most poorly understood in the CCO family.

Methods. By using as a reference the Chandra X-ray coordinates of RX J0822.0-4300 we performed deep optical observations in the $B, V$ and $I$ bands with the Very Large Telescope (VLT).

Results. We found no candidate optical counterpart within $3 \sigma$ of the computed Chandra X-ray position down to $5 \sigma$ limits of $B \sim 27.2$, $V \sim 26.9$, and $I \sim 25.6$, the deepest obtained in the optical band for this source.

Conclusions. These limits confirm the non-detection of a companion brighter than an M 5 dwarf. At the same time, they do not constrain optical emission from the neutron star surface, while emission from the magnetosphere would require a spectral break in the optical/IR.
\end{abstract}

Key words. stars: neutron - stars: individual: RX J0822.0-430

\section{Introduction}

Observations performed with the Einstein satellite lead to the discovery of puzzling X-ray sources in young ( 2-10 kyears) supernova remnants (SNRs), i.e. 1E 1613-5055 in RCW 103 (Tuohy \& Garmire 1980) and 1E 1207-5209 in PKS 1209-52 (Helfand \& Becker 1984). Their location, very close to the geometrical centre of the remnant, suggested that they were young neutron stars formed by the supernova explosions, despite no pulsations being detected at radio and X-ray wavelengths. About ten of these X-ray sources, dubbed central compact objects or CCOs (Pavlov et al. 2002), have been discovered to date. The CCO X-ray properties markedly differ from those of young rotation-powered INSs (see De Luca 2008, for a recent review). Their X-ray spectra are not purely magnetospheric, as is the case, e.g. for the Crab pulsar, but have strong thermal components. Furthermore, CCOs show no evidence of pulsar wind nebulae (PWN). X-ray pulsations have been firmly detected for two CCOs, 1E 1207-5209 ( $P=424 \mathrm{~ms}$; Zavlin et al. 2000) and CXOU J185238.6+004020 in Kes $79(P=105$ ms; Gotthelf et al. 2005). Interestingly, in both cases the measured upper limits on the period derivatives yielded spin down ages which are a factor of $\sim 10^{3}$ larger than the SNR age, as well as dipole

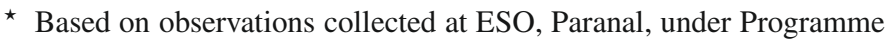
78.D-0706(A). magnetic fields $B<5 \times 10^{11} \mathrm{G}$ and rotational energy losses $\dot{E}<10^{32-33} \mathrm{erg} \mathrm{cm}^{-2} \mathrm{~s}^{-1}$ (Gotthelf \& Halpern 2007; Halpern et al. 2007). One possibility is that these CCOs were born spinning close to their present period and with very low magnetic fields, enabling polar cap accretion from a debris disc formed out of the supernova explosion. In the optical/infrared (IR), deep observations have been performed only for a handful of objects, mostly resulting in non-detections, which do not rule out the presence of a very low mass companion star ( $M$ type or later), or of a debris disc (see De Luca et al. 2008, and references therein).

One of the most interesting CCOs is that in the Puppis A SNR (3700 years). The X-ray source (RX J0822.0-4300), only hinted at in Einstein data (Petre et al. 1982), was then clearly detected by ROSAT (Petre et al. 1996). The source X-ray spectrum is predominantly thermal (Hui \& Becker 2006) and well fitted by a two-component blackbody model. The corresponding hydrogen column density $\left(N_{\mathrm{H}} \sim 4.54 \times 10^{21} \mathrm{~cm}^{-2}\right)$ is consistent with the $2.2 \mathrm{kpc}$ distance derived from neutral hydrogen observations of the SNR (Reynoso et al. 2003), which yield a 0.5$10 \mathrm{keV}$ X-ray luminosity of $L_{\mathrm{X}} \sim 2-5 \times 10^{33} \mathrm{erg} \mathrm{s}^{-1}$ for RX J0822.0-4300. Recently, a period at $\sim 112 \mathrm{~ms}$ has been reported from XMM-Newton observations by Gotthelf \& Halpern (2009). The first optical observations of RX J0822.0-4300 yielded upper limits of $B \sim 25$ and $R \sim 23.6$ on the source brightness (Petre et al. 1996) while new optical/IR observations (Wang et al. 2007) 
Table 1. Log of the VLT/FORS1 BVI observations of the Puppis A CCO (RX J0822.0-4300).

\begin{tabular}{lcccc}
\hline \hline yyyy-mm-dd & Filter & $T(\mathrm{~s})$ & Seeing & Airmass \\
\hline $2006-11-26$ & $I$ & 2800 & 0.88 & 1.09 \\
$2007-03-18$ & $B$ & 2800 & 0.98 & 1.06 \\
$2007-03-20$ & $B$ & 5600 & 0.84 & 1.07 \\
& $V$ & 2800 & 0.71 & 1.14 \\
$2007-03-23$ & $I$ & 8400 & 0.71 & 1.22 \\
$2007-03-24$ & $B$ & 2800 & 1.22 & 1.05 \\
$2007-03-25$ & $B$ & 2800 & 0.89 & 1.05 \\
\hline
\end{tabular}

Columns report the observing date, the filter name, the total integration time, the average seeing and airmass in each data set.

did not reveal any candidate counterpart down to $B \sim 26.5$ and $K_{\mathrm{S}} \sim 20.1$

Here we present deeper optical observations of RX J0822.0-4300, performed with the Very Large Telescope (VLT) of the European Southern Observatory (ESO). Observations and data reduction are described in Sect. 2, while the results are presented and discussed in Sects. 3 and 4, respectively.

\section{Observations}

\subsection{Observations and data reduction}

Optical imaging observations of RX J0822.0-4300 were performed in service mode with the VLT Kueyen telescope at the ESO Paranal observatory in November 2006 and March 2007 (see Table 1). The latter observations were performed right after the recoating of the telescope main mirror on March 2nd 2007. We used the FOcal Reducer/low dispersion Spectrograph (FORS1), a multi-mode camera for imaging and long-slit/multiobject spectroscopy. At the time of our observations the camera was still equipped with the original single chip, four port $2048 \times 2084$ Tektronik CCD detector. We selected the standard resolution mode, with a 0.2 pixel size, and a field of view of $6^{\prime} 8 \times 6^{\prime} 8$. Sequences of $560 \mathrm{~s}$ exposures were obtained through the Bessel $B, V$ and $I$ filters, for total integration times of 14000 , 2800, and $11200 \mathrm{~s}$, respectively. In order to avoid light contamination at the target position, bright stars in the field of view were masked using the FORSI occulting bars.

Observations were carried out with airmass better than $\sim 1.3$, and $\sim 0$ '. 8 seeing. Sky conditions were photometric (or close to) for all nights, with the exception of that of November 26th 2006. For this reason, and due to the limited amount of exposure time, in the following we do not make use of this observation. Bias, twilight flat-fields, and observations of standard stars from the Landolt fields (Landolt 1992) were obtained as part of the FORS1 science calibration plan. For the $V$-band observations no standard star observations were acquired on the night of March 21st. For photometric calibration purposes we thus used the closest-in-time available standard star observations, taken on March 10th.

Data were reduced (bias subtraction, flat-field correction, pre- and overscan trimming) using the ESO FORS1 data reduction pipeline ${ }^{1}$. We applied the photometric calibration using the night zero points available in the instrument data quality control database ${ }^{2}$. Airmass correction was applied using the extinction coefficients for the Paranal Observatory. For the March 21st

\footnotetext{
1 http://www.eso.org/observing/dfo/quality/FORS1/ pipeline

2 http://www. eso.org/observing/dfo/quality/
}

$V$-band data set the zero point trending plots ensure that the used closest-in-time zero point of March 10th, i.e. measured after the telescope recoating, does not introduce systematic effects in our photometric calibration. We converted the database zero points, computed in units of electrons/s, in units of ADU/s by applying the corresponding electrons-to-ADU conversion factors. For each dataset, we finally used the $I R A F$ tasks drizzle and imcombine to align and co-add all exposures taken with the same filter, as well as to mask bad pixels and filter cosmic ray hits.

\subsection{Astrometry calibration}

The astrometric calibration was computed on the FORS1 B-band image, taken as a reference, using the position and coordinates of 50 well-suited stars (e.g. not saturated, or too faint, or too close to the CCD edges, and evenly distributed in the field of view) selected from the GSC-2 version 2.3 (Lasker et al. 2008). The pixel coordinates of the GSC-2 stars were measured by fitting their intensity profiles with a Gaussian function using the dedicated tool of the Graphical Astronomy and Image Analysis (GAIA) interface ${ }^{3}$. The transformation between the detector and sky coordinates was then computed using the Starlink code ASTROM ${ }^{4}$ which is based on higher order polynomials and accounts for unmodeled CCD distorsions. The rms of our astrometric solution is $\delta r \approx 0.15$, accounting for the rms of the fit in the right ascension and declination components, to which we added in quadrature the uncertainty in the registration of the FORS1 image on the GSC-2 reference frame. Following Lattanzi et al. (1997), we estimated it as $\sqrt{3} \times \sigma_{\mathrm{GSC}} / \sqrt{N}_{s}$, where the $\sqrt{3}$ term accounts for the free parameters ( $x$-scale, $y$-scale, and rotation angle) in the astrometric fit, $\sigma_{\mathrm{GSC}}=0.3$ is the mean positional error of the GSC-2 coordinates (Lasker et al. 2008) and $N_{\mathrm{s}}$ is the number of stars used for the astrometric calibration. The uncertainty on the reference star centroids is below 0.'01 and was neglected. We also added in quadrature the 0 .' 15 uncertainty (Lasker et al. 2008) on the link of the GSC-2 to the International Celestial Reference Frame (ICRF). Thus, the overall accuracy of the FORS1 astrometry is $0.22(1 \sigma)$.

\section{Results}

As a reference for the RX J0822.0-4300 position we used the Chandra coordinates reported in Winkler \& Petre (2007), i.e. $\alpha(J 2000)=08^{\mathrm{h}} 21^{\mathrm{m}} 57.355^{\mathrm{s}}, \delta(J 2000)=-43^{\circ} 00^{\prime} 17^{\prime \prime} .17($ epoch 2005.32; $\mathrm{MJD}=53485)$, with a $1 \sigma$ uncertainty of \pm 0 ' 01 per each coordinate. To register the RX J0822.0-4300 position on our FORS1 B-band image (epoch 2007.23; MJD = 54184) we then accounted for its Chandra proper motion of $165 \pm$ 25 mas yr$^{-1}$ (Winkler \& Petre 2007), obtaining $\alpha(J 2000)=$ $08^{\mathrm{h}} 21^{\mathrm{m}} 57.309^{\mathrm{s}}, \delta(J 2000)=-43^{\circ} 00^{\prime} 17^{\prime \prime} .28$. We finally estimated the overall uncertainty on the RX J0822.0-4300 position at the epoch of our FORS1 observations by adding in quadrature the positional error on the Chandra coordinates at the reference epoch (0.'015), the coordinate uncertainty due to the proper motion extrapolation $\left(\sim 00^{\prime} 048\right)$, and the error of our astrometric calibration $(\sim 0.22)$. This yields to an overall uncertainty of 0.23 $(1 \sigma)$. We note that both the Chandra coordinates and the GSC-2 ones, used for the astrometric calibration of our FORS1 images, are all linked to the ICRF so that we exclude any systematic offset in our astrometry.

\footnotetext{
3 http://star-www.dur.ac.uk/ pdraper/gaia/gaia.html

${ }^{4}$ http://star-www.rl.ac.uk/Software/software.htm
} 


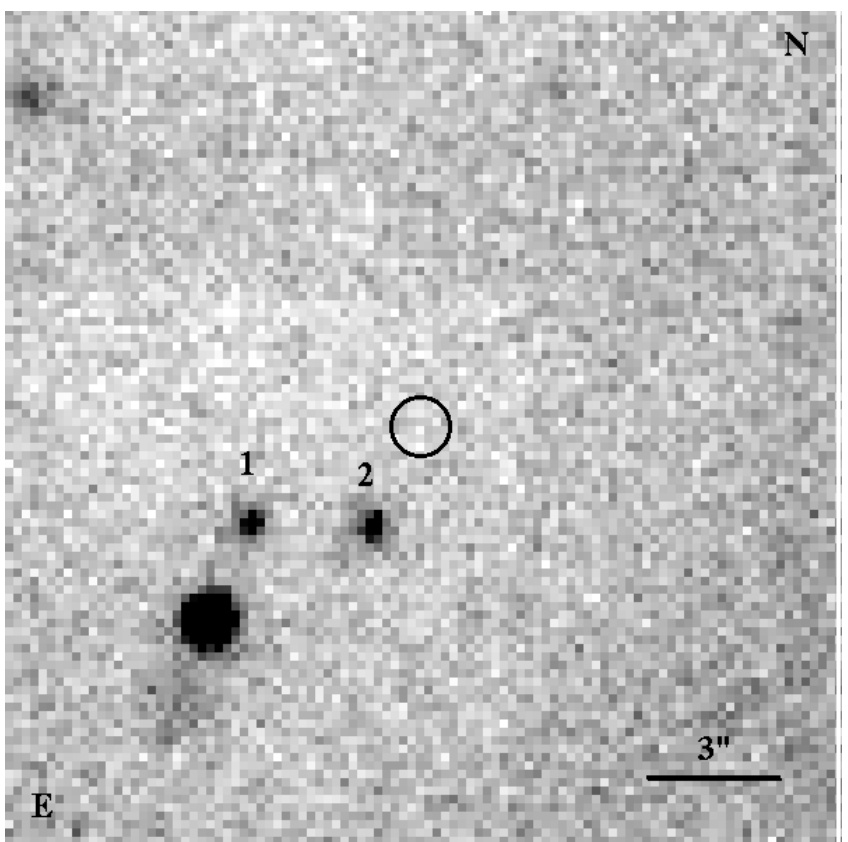

Fig. 1. $20^{\prime \prime} \times 20^{\prime \prime}$ cutout of the co-added FORS1 B-band image (14000 s) of the RX J0822.0-4300 field. The CCO position is marked by the circle ( 0 ' 7 radius, $3 \sigma$ confidence level). The two objects detected closest to the Chandra position, also detected in the optical images of Wang et al. (2007), are labelled.

Figure 1 shows the computed Chandra position of RX J0822.0-4300 overlaid on the co-added FORS1 B-band image. We note that our error circle is offset by $\sim 1$ '. 3 from that shown in Fig. 1 of Wang et al. (2007). This is due to the X-ray source proper motion between the epoch of our VLT observation (March 24th 2007) and the Chandra one (January 1st 2000) from which they derived the reference coordinates for their Magellan observation (April 5th 2003). No candidate counterpart is detected within a conservative $\sim 3 \sigma$ error circle. We have computed the flux of the field objects through customized aperture photometry. The closest objects, marked as $1(B=25.6)$ and $2(B=25.8)$ in Fig. 1, are detected at $\sim 22^{\prime \prime} 6$ and $4 . .7$ southeast of the $\mathrm{CCO}$ position, respectively. Their measured offests are well beyond any possible residual uncertainty in our astrometry, which rules out any association with the CCO. No candidate counterpart is detected either in the $V$-band image or in the I-band one. We thus conclude that the optical counterpart of the Puppis A CCO is unidentified. We estimated the detection limits of our images by extrapolating the fluxes of the faintest objects detected close to the RX J0822.0-4300 position. To this aim, we run the object detection using the SExtractor program (Bertin \& Arnouts 1996) and we computed their magnitudes through customized aperture photometry. We thus derived $5 \sigma$ upper limits on the optical brightness of the RX J0822.0-4300 counterpart of $B \sim 27.2, V \sim 26.9$, and $I \sim 25.6$.

\section{Discussion and conclusions}

Our BVI flux limits confirm the non-detection of a companion star earlier than an M 5 dwarf, as suggested by the previous optical and near/mid-IR flux upper limits of Wang et al. (2007). Similar conclusions were recently reported for other CCOs (see De Luca 2008, and references therein). The available upper limits can also be used to constrain the optical/IR spectrum from the neutron star or from a possible fallback disc. In Fig. 2 we plotted

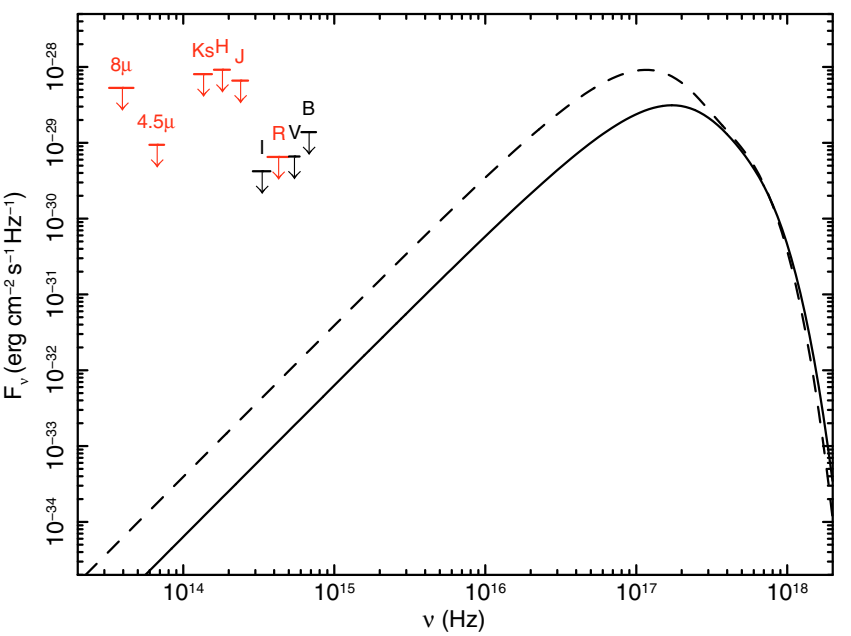

Fig. 2. Extrapolation in the optical/IR domain of the X-ray spectrum of RX J0822.0-4300. Both curves correspond to a double blackbody best-fit spectrum (Hui \& Becker 2006a) with (i) $T_{1}=2.61 \times 10^{6} \mathrm{~K}$, $T_{2}=5.04 \times 10^{6} \mathrm{~K}$, and emitting radii $R_{1}=3.29 \mathrm{~km}, R_{2}=0.75 \mathrm{~km}$ (solid line); and (ii) $T_{1}=1.87 \times 10^{6} \mathrm{~K}, T_{2}=4.58 \times 10^{6} \mathrm{~K}, R_{1}=10 \mathrm{~km}$, $R_{2}=1.09 \mathrm{~km}$ (dashed line). The extinction-corrected optical/IR flux upper limits (Wang et al. 2007, and present work) are plotted in black and red, respectively.

the extinction-corrected near/mid-IR and optical spectral flux upper limits together with the extrapolations of the best fits to the X-ray spectrum computed using double blackbody models with the hydrogen column density $N_{\mathrm{H}}$ left as a free parameter (Hui $\&$ Becker 2006). We computed the interstellar extinction correction in the $J H K_{\mathrm{s}}$ and $B V R I$ bands from the $N_{\mathrm{H}}$ derived from the X-ray spectral fits applying the relation of Predehl \& Schmitt (1995) and the extinction coefficients of Fitzpatrick (1999). For the mid-IR bands the interstellar extinction was computed from the $A_{V}$ using the relations of Chiar \& Tielens (2006). For simplicity, we plotted only the extinction-corrected spectral fluxes corresponding to an $N_{\mathrm{H}}=4.54 \times 10^{21} \mathrm{~cm}^{-2}$ derived from the first double blackbody spectral fit (see caption of Fig. 2). As seen, the $\mathrm{X}$-ray spectra extrapolations are at least two orders of magnitude below the optical/IR upper limits, even in the more favorable case of thermal emission from the bulk of the neutron star surface (dashed line in Fig. 2). On the other hand, for a blackbody plus power-law model $\left(T_{1}=8.57 \times 10^{6} \mathrm{~K} ; \Gamma=4.29\right)$ the $\mathrm{X}$ ray spectrum extrapolation falls about four orders or magnitudes above the optical/IR upper limits. Thus, we can not constrain optical/IR emission from the neutron star surface, while emission from the magnetosphere would obviously require a break in the source optical-to-X-ray power-law spectrum. We note that the spectral break implied by the broken power-law model fit to the X-ray spectrum $\left(\Gamma_{1}=3.61 ; \Gamma_{2}=5.29\right)$ would also overpredict the optical emission of RX J0822.0-4300 by an amount similar to the blackbody plus power-law model. However, detection of optical magnetospheric emission would require an energy output and an optical emission efficiency comparable to those of the Crab pulsar.

The presence of a fallback disc can not be ruled out by our data. For the assumed $0.5-10 \mathrm{keV}$ X-ray luminosity of $L_{X} \sim$ $2-5 \times 10^{33} \mathrm{erg} \mathrm{s}^{-1}$, the disc emission would be dominated by the reprocessed X-ray flux (Perna et al. 2000). The X-ray luminosity of RX J0822.0-4300 is within a factor of 2 from that of the CCO 1WGA J1713-3949 in G347.3-0.5, i.e. $L_{X} \sim 2.8 \times 10^{33} \mathrm{erg} \mathrm{s}^{1}$ at a distance of $1.3 \mathrm{kpc}$ (see Mignani et al. 2008, and references therein). This would imply, for the same set of disc model 
parameters, i.e. the disc inner and outer radii, the disc accretion rate, and the viewing geometry (Perna et al. 2000), a comparable disc IR luminosity. Thus, the optical/IR spectral flux upper limits of RX J0822.0-4300 are still consistent with the presence of, e.g. an undetected disc with outer radius smaller than $\approx 1 R_{\odot}$.

The present upper limits on the RX J0822.0-4300 optical emission can only be marginally improved with the $V L T$, or with other 8m-class telescopes. On the other hand, much deeper IR observations of RX J0822.0-4300 are still feasible with the VLT and could help to further constrain the presence of a fallback disc.

Acknowledgements. R.P.M. acknowledges STFC for support through its Rolling Grant programme. The authors thank Emanuela Pompei for support with the VLT observations and Rosalba Perna for useful comments.

\section{References}

Bertin, E., \& Arnouts, S. 1996, A\&AS, 117, 393

Chiar, J. E., \& Tielens, A. G. G. M. 2006, ApJ, 637, 774
De Luca, A. 2008, in Proc. of 40 Years of Pulsars: Millisecond Pulsars, Magnetars and More, AIP, 983, 311

Fitzpatrick, E. L. 1999, PASP, 111, 63

Gotthelf, E. V., \& Halpern, J. P. 2007, ApJ, 664, L35

Gotthelf, E. V., \& Halpern, J. P. 2009, ApJ, 695, L35

Gotthelf, E. V., Petre, R., \& Vasisht, G. 1999, ApJ, 514, L107

Halpern, J. P., Gotthelf, E. V., Camilo, F., \& Seward, F. D. 2007, ApJ, 665, 1304 Helfand, D. J., \& Becker, R. H. 1984, Nature, 307, 215

Hui, C. Y., \& Becker, W. 2006, A\&A, 454, 543

Landolt, A. 1992, AJ, 104, 340

Lasker, B., Lattanzi, M. G., McLean, B. J., et al. 2008, AJ, 136, 735

Lattanzi, M. G., Capetti, A., \& Macchetto, F. D. 1997, A\&A, 318, 997

Mignani, R. P., Zaggia, S., De Luca, A., et al. 2008, A\&A, 484, 457

Pavlov, G. G., Sanwal, D., Garmire, G. P., \& Zavlin, V. E. 2002, in Proc. of Neutron Stars in Supernova Remnants, ASP Conf. Ser., 271, 247

Petre, R., Kriss, G. A., Winkler, P. F., \& Canizares, C. R. 1982, ApJ, 258, 22 Petre, R., Becker, C. M., \& Winkler, P. F. 1996, ApJ, 465, L43

Perna, R., Hernquist, L., \& Narayan, R. 2000, ApJ, 541, 244

Predehl, P., \& Schmitt, J. H. M. M. 1995, A\&A, 293, 889

Reynoso, E. M., Green, A. J., Johnston, S., et al. 2003, MNRAS, 345, 671

Tuhoy, I., \& Garmire, G. 1980, ApJ, 239, L107

Wang, Z., Kaplan, D. L., Chakrabarty, D. 2007, ApJ, 655, 261

Winkler, P. F., \& Petre, R. 2007, ApJ, 670, 635

Zavlin, V. E., Pavlov, G. G., Sanwal, D., \& Trümper, J. 2000, ApJ, 540, L25 